\title{
Variations de composition et nature de la fraction insoluble des lactosérums industriels
}

\author{
F Saulnier ${ }^{1}$, F Ferrero ${ }^{1,2}$, JY Bottero ${ }^{3}$, G Linden ${ }^{1}$ \\ 1 Laboratoire de biochimie appliquée-associé à I'INRA, Université de Nancy I, \\ Faculté des sciences, BP 239, 54506 Vandœuvre-lès-Nancy; \\ 2 Lacto Sérum France, Baleycourt, BP 64, 55102 Verdun Cedex; \\ ${ }^{3}$ Laboratoire environnement et minéralurgie, École nationale supérieure de géologie, \\ rue du Doyen-Marcel-Roubault, BP 40, 54501 Vandœuvre-lès-Nancy, France
}

(Reçu le 20 mai 1994 ; accepté le 3 novembre 1994)

\begin{abstract}
Résumé - Les teneurs en calcium, citrate et phosphate des lactosérums doux et acides préconcentrés à $30 \%$ d'extrait sec, présentent de fortes variations. Le précipité obtenu lors de l'étape de préconcentration est constitué essentiellement de phosphate de calcium et de citrate de calcium. La nature exacte de ce citrate n'a pas pu être élucidée ; les analyses par diffraction des rayons $X$ et spectrométrie infra-rouge révèlent la présence de brushite dans ce précipité.
\end{abstract}

lactosérum / calcium / citrate / phosphate / brushite

Summary - Variation of the composition and nature of the insoluble precipitate from industrial wheys. In the industrial preconcentrated wheys ( $30 \%$ dry matter) important variations of calcium, citrate and phosphate concentrations are observed. By a simple method, which can be used in an industrial environment, the inorganic precipitate which appears during preconcentration has been analysed. It is composed essentially of calcium phosphate and calcium citrate. The exact nature of this citrate remains still unclear. $X$-ray diffraction and infra-red spectrometry reveal the presence of brushite in this precipitate.

whey / calcium / citrate / phosphate / brushite

\section{INTRODUCTION}

Les causes des différences de composition minérale et protéique des lactosérums sont en premier lieu à rattacher aux variations qualitatives des laits et, en second lieu, à la diversité des technologies fromagères (Roeper, 1971 ; Matthews, 1978 ; Huffman, 1988).

Le facteur saisonnier constitue la cause la plus importante de variation de la composition minéralo-protéique du lait ; il 
résulte des effets combinés de l'alimentation, des facteurs climatiques et du stade de lactation des vaches. Les variations de la composition minérale du lait ont déjà fait l'objet de nombreuses études. Les changements de la concentration saline en fonction du stade de lactation et du nombre de lactations ont été analysés par White et Davies, 1958 (cités par Holt et Muir, 1979). Barry et Rowland, 1953 (cités par les mêmes auteurs) ont trouvé une corrélation négative entre les concentrations en sodium, potassium, chlorures, et le contenu en lactose du lait. Les facteurs influençant les concentrations en calcium, en magnésium, en phosphate et en citrate sont mal connus. Holt et Muir (1979) ont enregistré des variations saisonnières importantes des teneurs en calcium du lait cru et mettent en évidence des corrélations entre les concentrations en calcium et citrate de la phase soluble.

Selon la technologie fromagère utilisée, les lactosérums obtenus peuvent être (doux) ou acides (lactiques). Le lactosérum doux est obtenu durant la fabrication des fromages à caractère "présure" après précipitation de la caséine et de la matière grasse du lait. II contient tous les constituants solubles au $\mathrm{pH}$ du lait non précipités par l'action des enzymes coagulantes. Blanc (1969) a donné la composition de différents lactosérums doux en la comparant avec celle des laits mis en œuvre. Les lactosérums acides sont issus de fabrications fromagères basées sur la coagulation isoélectrique ou de caséineries. Pour résumer, il existe autant de lactosérums que de technologies fromagères ; pour un même fromage, les lactosérums peuvent même différer selon l'usine. Ainsi, il est difficile de donner une composition minérale standard des lactosérums. Cependant, dans la littérature sont rapportées des compositions moyennes de lactosérums acides et doux (Hargrove et al, 1976; Webb, 1972).
Les minéraux et les acides organiques peuvent avoir une action sur la sensibilité thermique et la précipitation des protéines et des concentrés protéiques de lactosérums. Par exemple, l'addition de calcium à des concentrés protéiques ( 85 à $90 \%$ de protéines pour $100 \mathrm{~g}$ de matière sèche) à $\mathrm{pH}$ $6,60-6,90$, favorise la précipitation des protéines au chauffage (Hidalgo et Gamper, 1977). Les 4 cations majeurs (calcium, magnésium, sodium, potassium) se trouvent dans le lactosérum en présence de phosphates, citrates, chlorures, carbonates et lactates. Selon le degré de saturation, ces ions peuvent être totalement sous forme soluble ou en partie sous forme insoluble. D'une façon générale, il a été observé que les chlorures et les alcalins (potassium, sodium), sont dans les lactosérums complètement dissociés. En revanche, les anions phosphates, citrates, carbonates, lactates et les alcalino-terreux (calcium, magnésium) sont plus ou moins dissociés selon le $\mathrm{pH}$ du lactosérum.

Par une méthodologie simple, nous étudierons les fractions minérale et organique des lactosérums préconcentrés industriels et essayerons de déterminer la nature des sels insolubilisés.

\section{MATÉRIEL ET MÉTHODES}

\section{Échantillons de lactosérums}

Les lactosérums doux sont concentrés de 6 à $30 \%$ d'extrait sec (ES) sur les sites de production fromagère. Suivant leur origine et la valeur du $\mathrm{pH}$, ils sont triés et stockés à $30 \%$ d'ES, à une température inférieure à $10^{\circ} \mathrm{C}$. Les lactosérums préconcentrés doux proviennent de la SA Lacto Sérum France (Verdun, France), et ont des valeurs moyennes de $\mathrm{pH}$ comprises entre 5,80 et 6,20 .

Les lactosérums acides ( $\mathrm{pH}$ moyen de 4,60 ) sont issus de technologies au lait cru (pâtes molles), de technologies pâtes fraîches, de caséi- 
neries ; ils sont préconcentrés à $30 \%$ d'ES et collectés par la même entreprise.

\section{Détermination de l'insoluble pondéral}

Trente millilitres de lactosérum préchauffé à $40^{\circ} \mathrm{C}$ sont pesés et centrifugés $5 \mathrm{~min}$ à $2000 \mathrm{~g}$. Le surnageant et le culot sont pesés. Le résultat est validé quand l'écart entre 2 mesures est inférieur à $0,5 \%$ pour un même échantillon.

\section{Dosage des cations}

Le dosage des cations alcalins est réalisé par spectrométrie d'émission atomique et celui des cations alcalino-terreux est effectué par spectrométrie d'absorption atomique selon Linden et al (1970).

\section{Dosage des chlorures}

La teneur en chlorures est mesurée par titrage potentiométrique avec une solution de nitrate d'argent selon la norme de la Fédération internationale de laiterie (FIL) $n^{\circ} 88,1979$.

\section{Dosage du phosphore}

La teneur en phosphore est déterminée, après minéralisation par l'acide sulfurique en présence de peroxyde d'hydrogène, par la mesure au spectrophotomètre de l'absorbance due au complexe obtenu après traitement au molybdate de sodium et au sulfate d'hydrazinium. (Méthode de la FIL $\left.n^{\circ} 42,1967\right)$.

\section{Dosage de l'acide citrique}

La teneur en acide citrique est déterminée par réaction enzymatique. Après conversion de l'acide citrique en oxaloacétate et en acétate par une citrate lyase (EC 4,1.3.6), l'oxalate et son produit de décarboxylation, le pyruvate, sont réduits en Lmalate et en L-lactate en présence de malate déshydrogénase (EC 1.1.1.37) et de L-lactate déshydrogénase. Après réaction, l'absorbance à $340 \mathrm{~nm}$ de la $\mathrm{NADH}, \mathrm{H}^{+}$est mesurée (Boehringer, Mannheim, Allemagne).

\section{Détermination de la nature des structures cristallines}

Diffraction des rayons $X$ : diffractomètre JobinYvon Sigma 2080 (Saint-Louis, Ėtats-Unis) avec une longueur d'onde du rayonnement incident de $0,15406 \mathrm{~nm}$ et une intensité du faisceau de 6 à $20 \mathrm{~mA}$.

Spectroscopie infrarouge en mode transmission : après mélange de la poudre à analyser à du bromure de potassium dans une proportion de 1 à $2 \%$ de l'échantillon, des pastilles sont fabriquées à l'aide d'une presse hydraulique 0025 (Beckman instruments, Fullerton, États-Unis).

Spectroscopie infrarouge en mode réflexion: la poudre à analyser est mélangée à du bromure de potassium dans une proportion de $25 \%$. L'échantillon ainsi préparé est installé dans l'accessoire (Harrick) de réflexion. L'analyse est réalisée à l'aide d'un spectrophotomètre d'absorption infra-rouge à transmission de Fourier IFS 88 (Bruker, Billerica, États-Unis). Les spectres ont été obtenus en transmission et en réflexion.

\section{RÉSULTATS ET DISCUSSION}

\section{Variations des fractions minérale et organique des lactosérums industriels préconcentrés}

Les teneurs moyennes en citrate, phosphate et calcium de 17 lactosérums préconcentrés doux et acides de grands mélanges sont rapportées dans le tableau I. Les teneurs exprimées en molarités sont calculées en rapportant l'ES des lactosérums analysés à $30 \%$. En moyenne, les teneurs en citrate, phosphate et calcium des lactosérums doux sont respectivement de 30 , 66 et $49 \mathrm{mmol} / \mathrm{l}$, celles des lactosérums acides de 20, 92 et $111 \mathrm{mmol} / \mathrm{l}$. Ces der- 
Tableau I. Composition de 24 lactosérums préconcentrés doux et de 11 lactosérums préconcentrés acides.

Composition of 24 preconcentrated sweet wheys and 11 preconcentrated acid wheys.

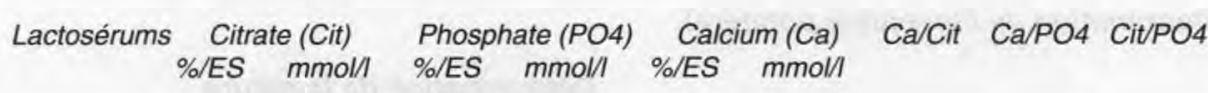

\begin{tabular}{|c|c|c|c|c|c|c|c|c|c|}
\hline \multicolumn{10}{|l|}{ Doux } \\
\hline Moyenne & 1,9 & 29,7 & 2,08 & 65,7 & 0,65 & 48,7 & 1,64 & 0,44 & 0,45 \\
\hline $\mathrm{CV} \%$ & 19,3 & 19,3 & 11 & 11 & 17 & 17 & & & \\
\hline \multicolumn{10}{|l|}{ Acides } \\
\hline Moyenne & 1,3 & 20,3 & 2,9 & 91,6 & 1,48 & 111 & 5,47 & 1,21 & 0,22 \\
\hline CV\% & 21 & 21 & 7 & 7 & 12,5 & 12,5 & & & \\
\hline
\end{tabular}

niers contiennent 2 fois plus de calcium que les lactosérums doux. Cette différence s'explique par la forte déminéralisation du caillé lors de la fabrication des produits laitiers à caractère lactique (pâte fraîche, pâte molle de fin d'égouttage etc...).

Quels que soient les lactosérums, les teneurs en calcium et en citrate varient beaucoup plus que celles des phosphates. Ces variations sont dues aux mélanges. En effet, dans une cuve de $100 \mathrm{~m}^{3}$ sont mélangés 4 à 20 lactosérums différents. Par conséquent, les coefficients de variation (CV) élevés (7 à 21\%) ne sont pas surprenants. Nous constatons de plus fortes variations sur les teneurs en calcium et en citrates. L'acide citrique est métabolisé par certains ferments utilisés en technologie laitière, lesquels se retrouvent et se développent dans le lactosérum s'il n'est pas rapidement refroidi.

Certains lactosérums acides proviennent de la phase d'égouttage ; par conséquent, lors de l'acidification en moule il y a une solubilisation du calcium, du magnésium et du phosphate qui se traduit par une augmentation des teneurs dans le lactosérum. En ce qui concerne le phosphate minéral, la solubilisation est totale vers $\mathrm{pH} 5,20$, tandis que pour le calcium et le magnésium il faut atteindre une valeur proche de $\mathrm{pH} 3,50$ (Le Graet et Brulé, 1993). II en résulte une plus forte variation du taux de calcium par rapport à celui du phosphate sans oublier la fluctuation des $\mathrm{pH}$ entre 4,30 à 5,30 .

Pour les lactosérums doux, de bonnes corrélations sont mises en évidence entre les taux de calcium et de citrate d'une part (exprimés en \%/ES) et le $\mathrm{pH}$ d'autre part. Les coefficients de corrélation sont respectivement de 0,86 et de 0,89 pour 17 échantillons. La teneur en citrate augmente avec le $\mathrm{pH}$ et la teneur en calcium diminue (résultats non publiés).

En résumé, la composition minérale des lactosérums préconcentrés industriels présente d'importantes variations, dues notamment aux différences technologiques ; la partie insoluble est importante et mérite une étude approfondie.

\section{Variations de l'insoluble pondéral des lactosérums}

L'insoluble pondéral déterminé après centrifugation à $2000 \mathrm{~g}$ pendant $5 \mathrm{~min}$ varie de 0 à $10 \%(\mathrm{~g} / 100 \mathrm{~g})$. Nous avons étudié la nature des sels insolubilisés et avons calculé leurs teneurs dans le culot par rapport à 
leurs teneurs initiales. Nous avons tracé les droites de régression linéaire de l'insoluble total en fonction des différentes espèces insolubilisées.

Pour les lactosérums doux (fig 1) et acides (fig 2), les teneurs en sodium, potassium, magnésium et chlorures dans la fraction insolubilisée suivent celles de l'insoluble total (pentes des droites de régression de l'ordre de 1,0). En revanche, le calcium, les citrates et les phosphates sont plus fortement insolubilisés : les pentes des droites de régression linéaire rapportées dans le tableau II sont respectivement de 0,37, 0,32 , et 0,63 , (cas des lactosérums doux) et de $0,15,0,13$, et 0,43 (cas des lactosérums acides). Ainsi, un lactosérum doux préconcentré ayant $10 \%$ d'insoluble total contient $27 \%$ de calcium, $16 \%$ de phosphate et $31 \%$ de citrate. L'insolubilité apparente du citrate est jusqu'à 3 fois supérieure à celle du phosphate dans les lactosérums acides et jusqu'à 2 fois dans les lactosérums doux.

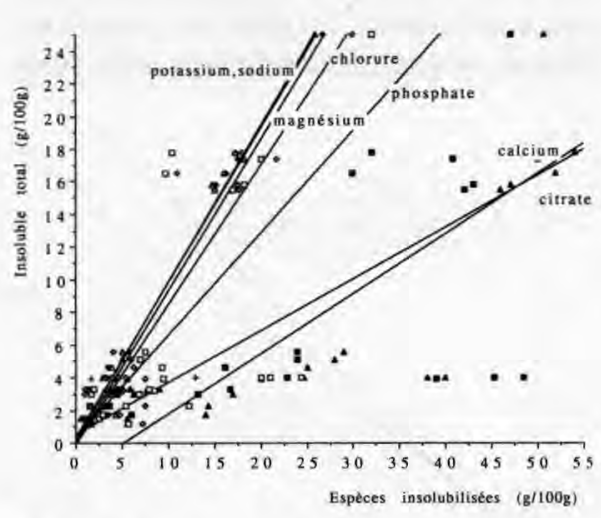

Fig 1. Évolution de l'insoluble total de 24 lactosérums préconcentrés doux en fonction de l'insolubilisation des différents ions dans le précipité : calcium ( $\mathbf{\Delta})$, citrate $(\boldsymbol{\square})$, phosphate $(\square)$, sodium $(\bullet)$ potassium $(\Delta)$, chlorure $(0)$, magnésium $(+)$. Evolution of total insolubilility in preconcentrated sweet wheys as a function of the insolubilization of different ions in the precipitate: calcium ( $\mathbf{\Delta})$,

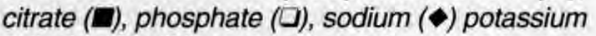
$(\Delta)$, chloride ( $(0)$, magnesium $(+)$.
Cette méthode simple nous a permis de mettre en évidence la présence d'un précipité minéral et de citrate dans l'insoluble total des lactosérums préconcentrés. L'insolubilisation apparente des minéraux se situe vers $10 \%$, même si certains d'entre eux sont en principe solubles (alcalins et chlorures). En revanche, l'insoluble total est inférieur aux insolubles apparents en citrates, phosphates et calcium pour les 2 types de lactosérums.

\section{Nature des sels précipités}

Les culots de centrifugation ( $2000 \mathrm{~g}, 5 \mathrm{~min}$ à $20^{\circ} \mathrm{C}$ ) des lactosérums doux et acides sont séparés par 3 lavages à l'eau déminéralisée et 3 centrifugations successives. La nature des ions est déterminée après dissolution des sels en phase acide $(\mathrm{HCl}$ $10 \mathrm{~N}$ ). Dans les lactosérums doux, seuls le citrate, le phosphate et le calcium sont retrouvés dans des proportions relatives

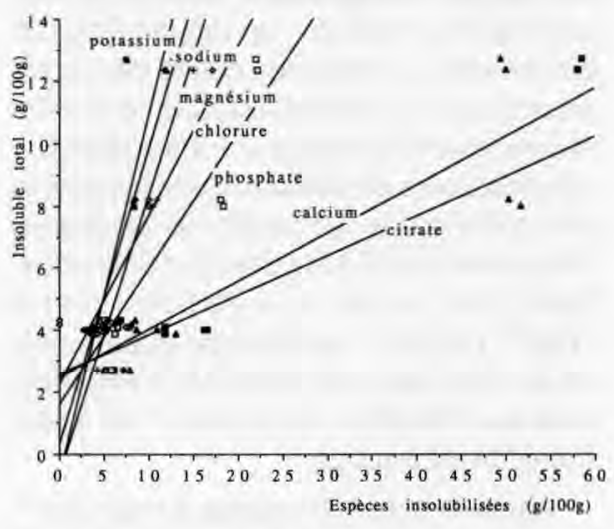

Fig 2. Évolution de l'insoluble total de 11 lactosérums préconcentrés acides en fonction de l'insolubilisation des différents ions dans le précipité : calcium $(\boldsymbol{\Delta})$, citrate $(\boldsymbol{\square})$, phosphate $(\square)$, sodium $(\bullet)$ potassium $(\Delta)$, chlorure $(0)$, magnésium $(+)$. Evolution of total insolubility in preconcentrated acid wheys as a function of the insolubilization of different ions in the precipitate: calcium ( $\mathbf{\Delta}$ ),

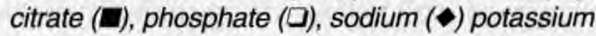
$(\Delta)$, chloride $(0)$, magnesium ( + ). 
Tableau II. Équation des droites de régression linéaire des figures $1(n=24)$ et $2(n=11)$. Linear curve fitting for figures $1(n=24)$ and $2(n=11)$.

Figure 2

\begin{tabular}{lcc} 
Espèce & \multicolumn{2}{c}{ Figure 1} \\
\cline { 2 - 3 } & $\begin{array}{c}\text { Équation } \\
\text { des droites }\end{array}$ & $\begin{array}{c}\text { Coefficient } \\
\text { de corrélation }\end{array}$
\end{tabular}

Espèce

$\begin{array}{lll}\text { Calcium } & Y=0,37 X-1,88 & 0,85 \\ \text { Citrate } & Y=0,32 X+0,55 & 0,71 \\ \text { Phosphate } & Y=0,63 X+0,28 & 0,71 \\ \text { Chlorure } & Y=0,85 X-0,05 & 0,95 \\ \text { Magnésium } & Y=0,93 X+0,02 & 0,97 \\ \text { Potassium } & Y=0,96 X+0,21 & 0,88 \\ \text { Sodium } & Y=0,95 X+0,33 & 0,99\end{array}$

$\begin{array}{ll}Y=0,15 X+2,48 & 0,9 \\ Y=0,13 X+2,57 & 0,89 \\ Y=0,43 X+1,63 & 0,95 \\ Y=0,54 X+2,25 & 0,91 \\ Y=0,89 X-0,59 & 0,98 \\ Y=1,15 X-0,66 & 0,88 \\ Y=0,94 X+0,37 & 0,74\end{array}$

allant de 10 à 80 (rapport molaire $\mathrm{PO}_{4} /$ Cit) et de 0,6 à 0,9 (rapport molaire $\mathrm{PO}_{4} / \mathrm{Ca}$ ). Les insolubilisations du phosphate et du calcium semblent donc liées et sont nettement supérieures à celles du citrate. Le phosphate de calcium est l'élément prépondérant de l'insoluble minéral. Les pentes des droites de régression linéaire du rapport $\mathrm{Ca} / \mathrm{Cit}$ en fonction du rapport $\mathrm{PO}_{4} / \mathrm{Cit}$, proches de 1,0 dans les culots des lactosérums doux ou acides (fig 3 ), le confirment et conduisent à penser à une insolubilisation de phosphates de calcium. Cette hypothèse est confirmée par les travaux de Madsen et Thorvardarson (1984) cités par Schmidt et Both (1987) et par ceux de Schmidt et al (1987). De plus, une analyse approfondie de la nature des sels précipités a été effectuée par diffraction des rayons $X$ et spectrométrie infra-rouge.

Après le troisième lavage à l'eau déminéralisée, les culots de centrifugation ont été lyophilisés avant d'être analysés.

La spectroscopie de diffraction des rayons $\mathrm{X}$, sensible aux caractères cristallins des solides, révèle pour les 2 types de lactosérums (figs $4 \mathrm{a}$ et $4 \mathrm{~b}$ ) une phase minérale dont le diagramme est proche de l'hydrogénophosphate de calcium dihydraté cristallisé : la brushite. La qualité des profils ne permet pas de conclure à la présence de brushite naturelle (fiche 11-293) ou synthétique (fiche 9-77) du fichier ASTM. Les diagrammes n'interfèrent pas avec celui du lactose $\alpha$ sous forme cristallisée réalisé par Saito (1988).

En spectroscopie infra-rouge, les spectres de la figure 5 confirment la présence de brushite. En effet, on retrouve les bandes caractéristiques de la brushite selon

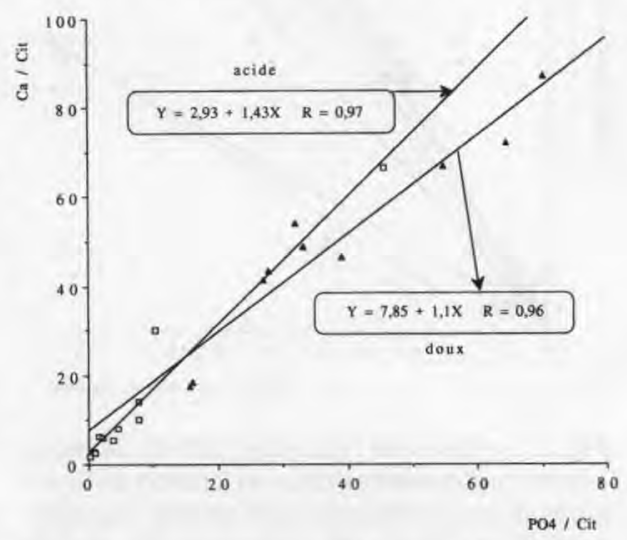

Fig 3. Évolution du rapport molaire $\mathrm{PO}_{4} / \mathrm{Cit}$ en fonction du rapport molaire $\mathrm{Ca} / \mathrm{Cit}$ pour les lactosérums préconcentrés doux $(\boldsymbol{\Delta})$ et acides $(\square)$. Variation of the molar $\mathrm{PO}_{4} /$ Cit ratio as a function of the molar Ca/Cit ratio for preconcentrated sweet wheys (4) and preconcentrated acid wheys (ㅁ). 
Ross (1974) (pointées par un *). L'analogie des profils en transmission et en réflexion suggère un état de surface très propre des 2 composés. Ajoutons que Chaplin (1984) a retrouvé dans de l'ultrafiltrat de lait du phosphate de calcium dont la solubilité est proche de celle de la brushite.

Les spectres des échantillons de lactosérums doux et acides indiquent la présence de composés organiques à 2925 $\mathrm{cm}^{-1}$, et à $2854 \mathrm{~cm}^{-1}$ donc probablement une chaîne alkyle qui doit comporter un nombre important de groupements $\mathrm{CH}_{2}$. Ces composés organiques ne sont pas uniquement localisés à la surface des cristaux mais mélangés avec eux puisqu'ils sont détectables en transmission et en réflexion. L'hypothèse la plus probable est la présence d'impuretés protéiques entraînées dans le culot de centrifugation. Le spectre révèle également des bandes caractéristiques du groupement carbonyle $\mathrm{C}=0$ à $1746 \mathrm{~cm}^{-1}$ et une contribution entre $1500 \mathrm{et}$ $1400 \mathrm{~cm}^{-1}$ pouvant être attribuée au groupement carboxylique d'acides aminés. II
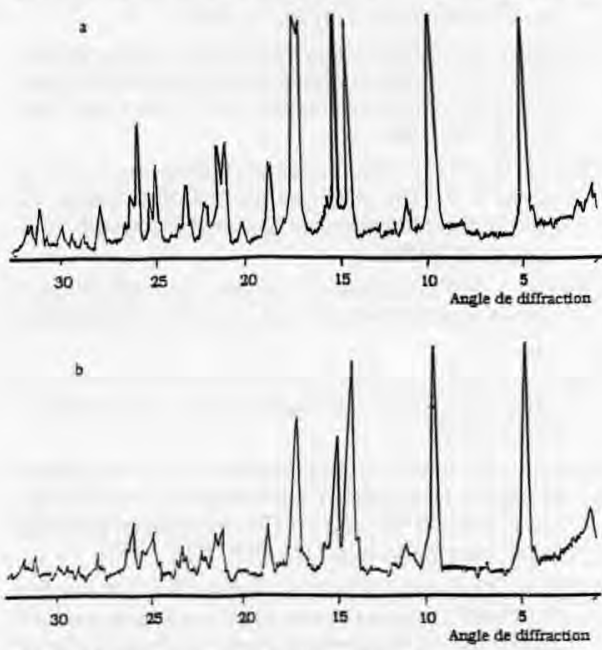

Fig 4. Diagramme de diffraction des rayons $X$ de précipités issus de lactosérums préconcentrés doux (a), et acide (b).

$X$ diffraction pattern of inorganic precipitates from preconcentrated sweet whey (a) and acid whey (b). peut s'agir de l'adsorption, sur les cristaux de phosphate de calcium, du fragment 1 105 ou 1-107 de la caséine $\beta$ par ses résidus phosphosérines, hypothèse avancée par Schmidt et al (1987) dans les lactosérums acides. Il a, selon les mêmes auteurs, un effet inhibiteur sur la croissance du phosphate de calcium. Ces résultats confirment les observations de Brulé et Fauquant (1981) et de Hiddinck et al (1981) qui démontrent une inhibition de la croissance du phosphate de calcium par certains composés du lait.

De surcroît, les intensités relatives des bandes des régions Amide I - Amide II des protéines d'une part $\left(1500-1700 \mathrm{~cm}^{-1}\right)$ et des phosphates (autour de $1000 \mathrm{~cm}^{-1}$ )

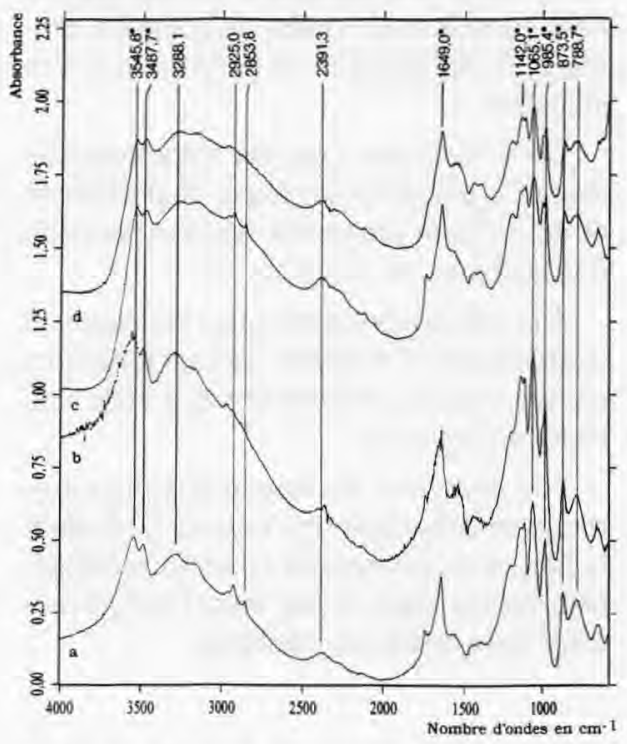

Fig 5. Spectres infra-rouges en transmission de précipités issus de lactosérums préconcentrés doux (a) ou acide (b) et en réflexion de précipités issus de lactosérums préconcentrés doux (c) ou acide (d). Les astérisques représentent les pics caractéristiques de la brushite.

Transmission infra-red spectra for inorganic precipitates from preconcentrated sweet (a) or acid (b) wheys and reflection infra-red spectra for inorganic precipitates from preconcentrated sweet (c) or acid (d) wheys. The * represent the characteristic peaks of brushite. 
d'autre part, ne permettent pas de conclure à une présence exclusive de brushite. La région de $3300 \mathrm{~cm}^{-1}$ semble bien corroborer ce type de conclusion, de même que le tableau 1.

\section{CONCLUSION}

Les fortes variations de la composition minérale des lactosérums industriels expliquent la formation d'une fraction insoluble plus ou moins importante lors de la préconcentration de ce coproduit.

L'insoluble minéral et citrique des lactosérums a été récupéré par centrifugation ; cette méthode simple a été choisie car elle peut constituer un indice de solubilité pratique et rapide pour un contrôle sur site industriel.

Cette méthode a permis notamment de révéler la présence de citrate, de phosphate et de calcium précipités dès la réception des lactosérums à l'usine.

À la préconcentration et au stockage, la déstabilisation minérale des lactosérums acides est plus importante que celle des lactosérums doux.

Les analyses par spectroscopie infrarouge et diffraction des rayons $X$ révèlent la nature du phosphate cristallin précipité. Néanmoins, elles ne permettent pas de préciser celle du citrate insoluble.

\section{REMERCIEMENTS}

Ce travail a été réalisé grâce à une aide de la mission Recherche de la région Lorraine et l'Association nationale pour la recherche technique (ANRT). Nous remercions F Thomas pour ses précieux conseils apportés dans la rédaction de cette publication.

\section{REFERENCES}

Blanc B (1969) Molke und daraus hergestellte erzeugnisse, aspekte der zusammensetzung und ernahrung des menschen. IDF-Seminar on whey processing and utilization. Weihenstephan-Germany, 11-13. Novembre 1969

Brulé G, Fauquant J (1981) Mineral balance in skim milk and retentate: effect of physicochemical characteristics of aqueous phase. J Dairy Res 48, 91-97

Chaplin LC (1984) Studies on micellar calcium phosphate: composition and apparent solubility product in milk over a wide $\mathrm{pH}$ range. J Dairy Res 51, 251-257

Hargrove RE, McDonough FE, La Croix DE, Alford JA (1976) Production and properties of deproteinized whey powders. J Dairy Sci 59, 25-33

Hidalgo J, Gamper E (1977) Solubility and heat stability of whey protein concentrates. I Dairy Sci60, 15151518

Hiddinck J, De Boer R, Nooy PFC (1981) Effect of various pretreatments on the ultrafiltration of sweet cheese whey at about $55^{\circ} \mathrm{C}$. Milchwissenschaft $36,657-663$

Holt C, Muir D (1979) Inorganic constituents of milk: 1. Correlation of soluble calcium with citrate in bovine milk. J Dairy Res 46, 433-439

Huffman LM (1988) Effect of heat treatment on the composition of whey protein concentrate. $N Z$ J Dairy Sci Technol 23, 337-344

Le Graet Y, Brulé G (1993) Les équilibres minéraux du lait: influence du $\mathrm{pH}$ et de la force ionique. Lait 73, 51-60

Linden G, Gohier B, Alais C, Tarodo de la Fuente B (1970) Essais de dessalification des produits laitiers par électrodialyse. Lait 50, 511-523

Matthews ME (1978) Compositions of rennet, sulphuric and lactic casein wheys and of sulphuric and lactic whey protein concentrates. NZJ Dairy Sci Technol 13, 149-156

Ross S D (1974) Phosphates and other oxyanions of group 5. In: The infra-red spectra of minerals (VC Farmer Ed) Mineralogical Society Monograph, London, 4, 383-422

Roeper J (1971) Seasonal changes in composition of rennet and acid wheys. N Z J Dairy Sci Technol 6 , $112-114$

Saito Z (1988) Lactose crystallization in commercial whey powders and in dried lactose. Food Microstruc 7, 75-81

Schmidt DG, Both P (1987) Studies on the precipitation of calcium phosphate. 1. Experiments in the $\mathrm{pH}$ range 5.3 to 6.8 at $25^{\circ} \mathrm{C}$ and $50^{\circ} \mathrm{C}$ in the absence of additives. Neth Milk Dairy J 41, 105-120

Schmidt DG, Both P, Visser S, Slangen KJ, Van Rooijen PJ (1987) Studies in the precipitation of calcium phosphate : 2. Experiments in the $\mathrm{pH}$ range 7.3 to 5.8 at $25^{\circ} \mathrm{C}$ and $50^{\circ} \mathrm{C}$ in the presence of additives. Neth Milk Dairy J 41, 121-136

Webb BH (1972) Recycling whey for profitable uses. Am Dairy Rev 34, 32A-32D 